Article

\title{
Graphene-Based Biosensors for Detection of Composite Vibrational Fingerprints in the Mid-Infrared Region
}

\author{
Yijun Cai ${ }^{1}$, Yanfen Hang ${ }^{2}$, Yuanguo Zhou ${ }^{2, *} \mathbb{C}$, Jinfeng Zhu ${ }^{3}{ }^{\mathbb{D}}$, Jingwen Yang ${ }^{3}$ and \\ Xuanyu Wang ${ }^{1}$ \\ 1 Fujian Provincial Key Laboratory of Optoelectronic Technology and Devices, Xiamen University of \\ Technology, Xiamen 361024, China; yijuncai@foxmail.com (Y.C.); wxyx815@163.com (X.W.) \\ 2 College of Communication and Information Engineering, Xi'an University of Science and Technology, \\ Xi'an 710054, China; yanfenhang@foxmail.com \\ 3 Department of Electronic Science, Xiamen University, Xiamen 361005, China; \\ nanoantenna@hotmail.com (J.Z.); 35320172200252@stu.xmu.edu.cn (J.Y.) \\ * Correspondence: wingkoo@foxmail.com; Tel.: +86-029-8558-3165
}

Received: 22 August 2019; Accepted: 17 October 2019; Published: 20 October 2019

\begin{abstract}
In this study, a label-free multi-resonant graphene-based biosensor with periodic graphene nanoribbons is proposed for detection of composite vibrational fingerprints in the mid-infrared range. The multiple vibrational signals of biomolecules are simultaneously enhanced and detected by different resonances in the transmission spectrum. Each of the transmission dips can be independently tuned by altering the gating voltage applied on the corresponding graphene nanoribbon. Geometric parameters are investigated and optimized to obtain excellent sensing performance. Limit of detection is also evaluated in an approximation way. Besides, the biosensor can operate in a wide range of incident angles. Electric field intensity distributions are depicted to reveal the physical insight. Moreover, another biosensor based on periodic graphene nanodisks is further proposed, whose performance is insensitive to the polarization of incidence. Our research may have a potential for designing graphene-based biosensor used in many promising bioanalytical and pharmaceutical applications.
\end{abstract}

Keywords: label-free biosensors; metasurface; graphene; surface plasmons; nanophotonics

\section{Introduction}

Surface plasmon resonance (SPR) biosensors have received tremendous attention over the past decades due to their label-free sensing ability [1,2]. They utilize surface plasmon polariton (SPP) waves to detect the refractive index (RI) change in the sensing surface produced by the alteration of biomolecule concentration. A variation in the propagation constant of SPP caused by the change of RI can be optically measured for inverse calculation of biomolecular RI. Nevertheless, in order to identify the species of biomolecule, roughly probing the change of RI in the biological processes is not sufficient. Characterization of biomolecules through their vibrational properties is also necessary to differentiate constituent biomolecular species. Vibrational fingerprints relating with the valuable molecular information are mainly located at the mid-infrared range, which uniquely identify the biomolecules in a multitude of biological processes. Infrared spectroscopy is widely used as a nondestructive label-free technique to access these vibrational fingerprints and provide exquisite biochemical information in bulk materials. However, vibrational signals in minute amounts of analytes are quite weak due to the mismatch between nanometric size of biomolecules $(<10 \mathrm{~nm})$ and the mid-infrared wavelengths $(2$ to $6 \mu \mathrm{m}$ ). To solve this problem, resonant metallic nanoantennas are commonly used to enhance light-matter interaction due to highly concentrated and enhanced infrared 
near fields [3-7], which is also called surface-enhanced infrared absorption (SEIRA). Although the resonant frequency can be tuned by adjusting the geometrical size of nanoantennas, biomolecules with spectrally separated vibrational bands usually require dual- or multiband plasmonic structures to minimize the number of false positive results [8,9]. On the other hand, to distinguish multiple analytes in heterogeneous biological samples, several multi-resonant infrared metallic or dielectric metasurfaces have been developed to simultaneously detecting the composite vibrational signals of different biomolecules [10-12].

Although the multi-resonant biosensors based on traditional materials can probe biomolecules in a relatively wide frequency range, their detected bands cannot be dynamically tuned to the designing frequencies after the fabrication process. To realize the postfabrication tuning, graphene has been taken into account as a promising candidate for biosensors. Graphene is a two-dimensional material with carbon atoms arranged in a honeycomb lattice $[13,14]$. Graphene-based photonic devices have attracted great interest due to its exceptional electrical and optical properties [15-18]. Optical biosensors based on graphene possess the advantage of tunable spectral selectivity owing to the tunability of graphene conductivity by electrostatic gating. Besides, the strong spatial light confinement in graphene also contributes to an enhanced sensitivity in the detection of RI changes and vibrational signals. In 2004, researchers from IBM Corporation utilized graphene nanoribbons to detect surface-adsorbed thin films of polymer for the first time [19]. They further investigated the coupling between graphene plasmons and the vibrations of solid- and gas-phase molecules [20]. Rodrigo et al. proposed a plasmonic graphene-based biosensor to identify the vibrational fingerprints of protein [21]. They experimentally demonstrated that dynamic resonance tuning could be achieved by varying the gating voltage and vibrational signals were significantly enhanced compared with gold nanoantennas. Hu et al. successively reported thin polymers sensing and gas identification with graphene plasmon, utilizing $\mathrm{CaF}_{2}$ nanofilm instead of $\mathrm{SiO}_{2}$ to avoid the plasmon-phonon hybridization [22,23]. Zhu et al. designed a hybrid metasurface with suspended graphene and gold nanoantennas, while the nanoantennas were deposited close enough (about $10 \mathrm{~nm}$ ), ultrasensitive biosensing ability was achieved to probe low-molecular-weight analytes [24]. The above reported graphene-based biosensors showed higher sensitivity and detection limit than traditional biosensors. However, they all suffered from the problem of single resonant property, which limited their applications in detecting composite vibrational fingerprints simultaneously.

In this paper, a label-free multi-resonant graphene-based biosensor (MRGB) is numerically proposed for detection of composite vibrational fingerprints in the mid-infrared region. Compared with the previous structures [18-24], the proposed biosensor requires simpler fabrication techniques. Each of the plasmonic resonances in transmission spectrum can be independently tuned by gating voltages to overlap with the vibrational fingerprints of different biomolecules at designing frequencies, leading to a wide spectral detection range. The influences of geometric parameters and incident angles on the performance of MRGB are discussed. Moreover, MRGB with graphene nanodisks are further designed to guarantee superior sensing ability under incident light with different polarizations.

\section{Modeling and Methods}

In order to realize a biosensor for detection of composite vibrational fingerprints, we utilize periodic graphene nanoribbons (GNRs) as a multi-resonant metasurface as plotted in Figure 1. GNRs can be grown by chemical vapor deposition and wet-transferred to the surface of $\mathrm{SiO}_{2}$ dielectric layer, while $\mathrm{Si}$ is chosen as the substrate to support this device. GNRs with different widths can be exposed using electron beam lithography. Wider GNRs are connected to the metallic electrode at the right far ends as the top contact, while narrower GNRs are connected to the metallic electrode at the left far ends as another top contact. The doped $\mathrm{Si}$ substrate is used as the bottom contact. Two bias voltages, $V_{g 1}$ and $V_{g 2}$, are applied on the interdigitated GNRs to control the Fermi energies $E_{F 1}$ and $E_{F 2}$ of GNRs separately. We assume that a mid-infrared plane wave illuminates on the top surface of MRGB, interacting with the graphene metasurface and the adsorbed biomolecules. The interaction 
is investigated using COMSOL Multiphysics, which solves Maxwell Equations with finite element method (FEM) in frequency domain. Floquet periodicity is chosen as boundary condition in both $x$ - and $y$-directions. Tetrahedral meshes are used in the entire domain. The transmittance of MRGB can be expressed as $T=\left|S_{21}\right|^{2}$, where $S_{21}$ can be directly obtained from the user-defined ports in the $z$-directions.
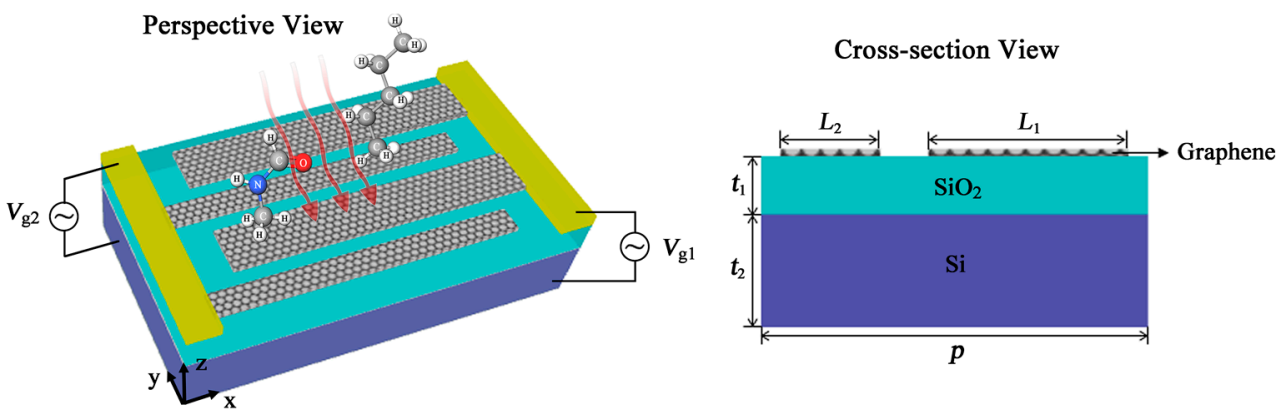

Figure 1. Schematic of multi-resonant graphene-based biosensor (MRGB). $L_{1}$ and $L_{2}\left(L_{1}>L_{2}\right)$ denote the widths of the wider and narrower graphene nanoribbons, respectively. $t_{1}$ and $t_{2}$ are the thicknesses of $\mathrm{SiO}_{2}$ and $\mathrm{Si}$. $p$ is the periodic graphene nanoribbons (GNRs) array pitch.

The permittivity of $\mathrm{SiO}_{2}$ and $\mathrm{Si}$ used in the simulation are chosen from Ref. [25]. In order to reduce the mesh number and improve the computational efficiency in simulation, we assume graphene as a $2 \mathrm{D}$ conductive surface without thickness, instead of a 3D bulk volume. This assumption has already been proved to be effective and efficient in the simulation of graphene-based devices before [26]. The surface conductivity of graphene can be calculated according to the well-known Kubo formulas [27]:

$$
\begin{gathered}
\sigma\left(\omega, E_{F}, \Gamma, T\right)=\sigma_{\text {intra }}+\sigma_{\text {inter }} \\
\sigma_{\text {intra }}=\frac{j e^{2}}{\pi \hbar^{2}(\omega-j 2 \Gamma)} \int_{0}^{\infty} \xi\left(\frac{\partial f_{d}\left(\xi, E_{F}, T\right)}{\partial \xi}-\frac{\partial f_{d}\left(-\xi, E_{F}, T\right)}{\partial \xi}\right) d \xi \\
\sigma_{\text {inter }}=-\frac{j e^{2}(\omega-j 2 \Gamma)}{\pi \hbar^{2}} \int_{0}^{\infty} \frac{f_{d}\left(-\xi, E_{F}, T\right)-f_{d}\left(\xi, E_{F}, T\right)}{(\omega-j 2 \Gamma)^{2}-4(\xi / \hbar)^{2}} d \xi \\
f_{d}\left(\xi, E_{F}, T\right)=\left(e^{\left(\xi-E_{F}\right) / k_{B} T}+1\right)^{-1}
\end{gathered}
$$

Here, $\sigma_{\text {intra }}$ and $\sigma_{\text {inter }}$ are the intraband and interband contributions, respectively. $\omega$ is the angular frequency, $E_{F}$ denotes the Fermi energy, $\Gamma$ is the scattering rate with $\Gamma=1 /(2 \tau), \tau$ is the relaxation time of electron-phonon, $T$ is the Kelvin temperature, $\hbar$ is the reduced Planck constant, $k_{B}$ is the Boltzmann constant, $e$ and $\xi$ are the electron charge and energy, $f_{d}\left(\xi, E_{F}, T\right)$ is the Fermi-Dirac distribution.

In the mid-infrared region, the intraband transition dominates the light-graphene interaction since the interband transition can be ignored. When $T$ is assumed to be $300 \mathrm{~K}$, Kubo formulas can be simplified as follows:

$$
\sigma_{g}=\frac{i e^{2} E_{F}}{\pi \hbar^{2}\left(\omega+i \tau^{-1}\right)}
$$

The realistic value of $\tau$ for graphene grown on the $\mathrm{SiO}_{2}$ substrate is usually smaller than $100 \mathrm{fs}$. To guarantee the validity and reliability of our simulation model, we choose $\tau$ as 15 fs according to the experimental work form Ref. [21], so the surface conductivity of graphene is determined by the Fermi energy $E_{F}$ and angular frequency $\omega$. An approximate closed-form expression between $E_{F}$ and gating voltage $V_{\text {bias }}$, is given by [28] as Equation (6):

$$
E_{F}=\hbar v_{f} \sqrt{\frac{\pi \varepsilon_{0} \varepsilon_{\mathrm{r}} V_{\text {bias }}}{e D}}
$$


where $\varepsilon_{0}$ is the permittivity of free space, $\varepsilon_{r}$ and $D$ are the equivalent relative permittivity and the thickness of insulating layers, respectively.

To demonstrate the detection ability for composite vibrational fingerprints of multiple biomolecules, we assume the protein and lipid molecules in the simulation as a thin layer with $8 \mathrm{~nm}$ thickness according to the previous experimental work [21], whose relative permittivity can be calculated by the Lorentzian series as:

$$
\varepsilon_{m}(\omega)=n_{\infty}^{2}+\sum_{k=1}^{N} \frac{S_{k}^{2}}{\omega_{k}^{2}-\omega^{2}-i \omega \gamma_{k}}
$$

The symbol $N$ denotes the number of oscillators, which is assumed as 3 relating to the corresponding parameters. The main vibrational fingerprints of proteins are amide I and II bands (about $1668 \mathrm{~cm}^{-1}$ and $1532 \mathrm{~cm}^{-1}$ ), while the $\mathrm{CH}_{2}$ band (about $2900 \mathrm{~cm}^{-1}$ ) is dominated in lipid molecules. Therefore, parameters are chosen as $\omega_{1}=1668 \mathrm{~cm}^{-1}, \omega_{2}=1532 \mathrm{~cm}^{-1}, \omega_{3}=2900 \mathrm{~cm}^{-1}, \gamma_{1}=78.1 \mathrm{~cm}^{-1}, \gamma_{2}=101 \mathrm{~cm}^{-1}$, $\gamma_{3}=111 \mathrm{~cm}^{-1}, S_{1}=213 \mathrm{~cm}^{-1}, S_{2}=200 \mathrm{~cm}^{-1}, S_{3}=230 \mathrm{~cm}^{-1}, n_{\infty}^{2}=2.08$, according to the experiment works from Refs. [11,21,29].

In our simulations, the exact sensitivity of MRGB is difficult to calculate due to the rough approximation of the biomolecules. However, the changing law of its sensitivity can be derived using the enhancement factor $(E F)$, which can be expressed as [4]:

$$
E F=\frac{I_{\text {SEIRA }}}{I_{0}} \times \frac{A_{0}}{A_{\text {SEIRA }}}
$$

Here, $I_{0}$ denotes the unenhanced signal strength, $A_{0}$ and $A_{\text {SEIRA }}$ are the areas covered with molecules in reference and SEIRA measurements. ISEIRA is the enhanced signal strength that can be obtained from the baseline-corrected vibrational signal. For our simulation, variation of any parameter has no effect on $I_{0}, A_{0}$ and $A_{\text {SEIRA }}$. Therefore, the changing law of sensitivity is directly related to the variation rule of $I_{\text {SEIRA }}$, which can be approximately observed from the simulated transmittance spectra.

\section{Results and Discussion}

Transmittance spectra of MRGB under mid-infrared incidence are plotted in Figure 2 to investigate the detection capacity for composite vibrational fingerprints of multiple biomolecules. The geometric parameters are $L_{1}=40 \mathrm{~nm}, L_{2}=20 \mathrm{~nm}, t_{1}=280 \mathrm{~nm}, t_{2}=500 \mathrm{~nm}$, and $p=80 \mathrm{~nm}$. Two obvious transmission dips are observed in the spectra under TMincidence, which is able to induce the electrons to vibrate in the finite width of GNRs due to its electric field direction. In contrast, TE incidence could not excite the plasmonic resonance in GNRs because of its electric field parallel to the nanoribbons. Hence, we focus on the sensing performance of MRGB with TM incidence in the following analysis.

In Figure 2a, by sweeping $E_{F 1}$ from $0.40 \mathrm{eV}$ to $0.60 \mathrm{eV}$, the first transmission dip of MRGB without protein and lipid is electrostatically tuned from $1525 \mathrm{~cm}^{-1}$ to $1875 \mathrm{~cm}^{-1}$, while the second resonance frequency is located around $3300 \mathrm{~cm}^{-1}$, with $E_{F 2}$ fixed at $0.85 \mathrm{eV}$. After the adsorption of protein and lipid molecules, the spectra are red-shifted due to the RI change at the surface of MRGB. Although the thickness of biomolecule layer is only several nanometers, the detected frequency shifts still exceed $350 \mathrm{~cm}^{-1}$, which is better than Ref. [21]. Besides, two vibrational fingerprints at $1668 \mathrm{~cm}^{-1}$ and $1532 \mathrm{~cm}^{-1}$ are almost undetectable as the resonance dip is far from them (e.g., for $E_{F 1}=0.40 \mathrm{eV}$ ). As the spectral overlap increases, the vibrational signals become progressively more intense (e.g., for $E_{F 1}=0.60 \mathrm{eV}$ ), whose spectral positions are in accordance with the amide I and II bands, respectively. 
(a)

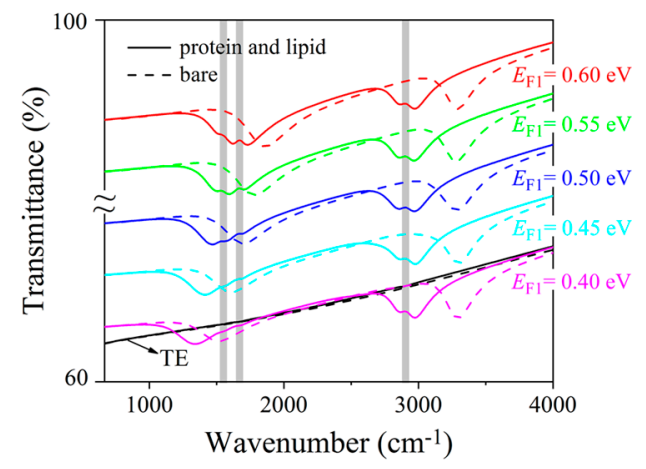

(b)

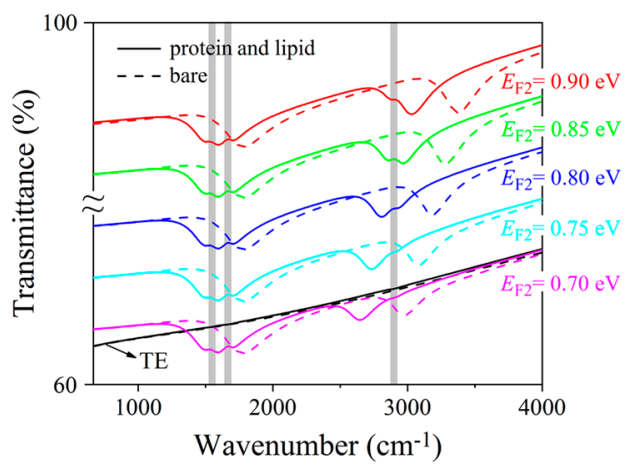

Figure 2. Transmittance spectra of MRGB under transverse electric (TE) (black curves) and transverse magnetic (TM) (colored curves) mid-infrared incidence while sweeping $E_{F 1}(\mathbf{a})$ and $E_{F 2}(\mathbf{b})$ of graphene, with (solid curves) and without (dashed curves) protein and lipid molecules. Gray vertical bars represent vibrational bands of protein and lipid molecules. Curve with larger $E_{F}$ is translated upward with $6 \%$ in order to demonstrate the spectral shift clearly.

In Figure $2 \mathrm{~b}$, the second resonant transmission dip is tuned by gating voltage $V_{g 2}$ to sweep over the lipid vibrational band independently. When $E_{F 1}=0.55 \mathrm{eV}$ and $E_{F 2}=0.70 \mathrm{eV}$, the vibrational bands in protein are easily detected attributed to the first transmission dip. However, the lipid vibrational band is almost undetectable since it is far from the second transmission dip. As $E_{F 2}$ increases to $0.85 \mathrm{eV}$, the lipid sensing is achieved by narrow peak appearing at $2900 \mathrm{~cm}^{-1}$ corresponding to the $\mathrm{CH}_{2}$ band. It unambiguously reveals the presence of the lipid compounds in a chemically specific manner. If $E_{F 2}$ continues increasing to $0.90 \mathrm{eV}$, the detected lipid vibrational signal becomes weakened as the second resonant dip moves away to larger frequencies gradually.

Therefore, the proposed MRGB can not only work as a refractive index biosensor by detecting a plasmon resonance spectra shift, but also identify composite vibrational fingerprints in multiple biomolecules simultaneously due to the resonant coupling between plasmons and molecular vibrations [30]. Moreover, it possesses the independent tuning ability for individual plasmonic resonance, which is significant and flexible in practical sensing applications.

We depict the electric field distributions in Figure 3 to illustrate the resonant property of MRGB with protein and lipid molecules. As can be seen from Figure 2, when $E_{F 1}=0.55 \mathrm{eV}$ and $E_{F 2}=0.85 \mathrm{eV}$, the transmission spectra show two obvious dips around $1600 \mathrm{~cm}^{-1}$ and $2967 \mathrm{~cm}^{-1}$. At $k=1600 \mathrm{~cm}^{-1}$, the enhanced electric field is dramatically concentrated along the edges of the wider GNRs as shown in Figure 3a. This is attributed to the fact that the incident TM mid-infrared light can excite electrons of graphene to oscillate in the finite width of nanoribbon, inducing the localized surface plasmon resonance (LSPR) in the wider GNRs [31,32]. Similarly, at $k=2967 \mathrm{~cm}^{-1}$ as shown in Figure 3c, plasmonic hotspots are generated at the edges of the narrower GNRs. The localized resonance originates from the electric dipole surrounding the edges of narrower GNRs and contributes to the corresponding transmission dip in the spectrum. Compared with gold nanoribbons, a higher field confinement for similar infrared-frequency plasmons is observed due to the atomic thickness of graphene as demonstrated in Ref. [21]. Consequently, a much larger spatial overlap between the plasmonic near field and biomolecules occurs, leading to a better sensitivity than biosensor based on metallic nanoantennas [21]. 

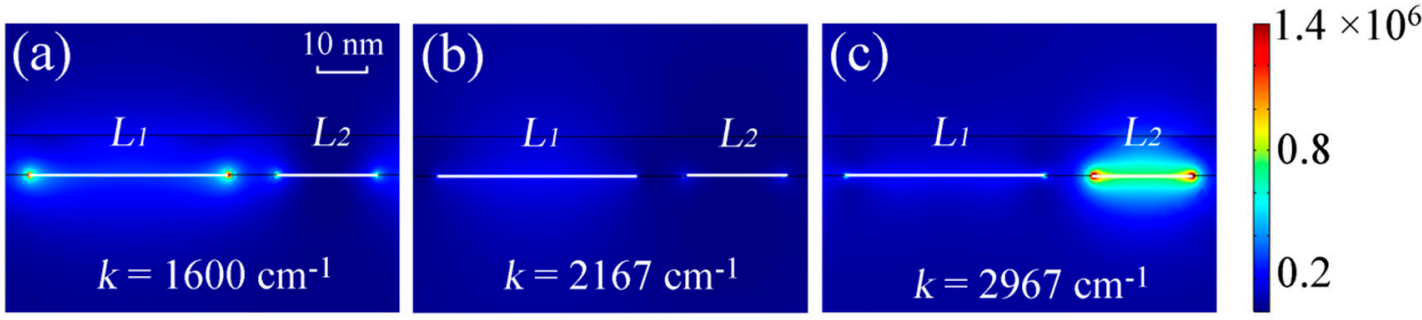

Figure 3. Side view of electric field distributions of MRGB with protein and lipid at some specific wavenumbers for (a) $k=1600 \mathrm{~cm}^{-1}$, (b) $k=2167 \mathrm{~cm}^{-1}$, (c) $k=2967 \mathrm{~cm}^{-1}$ under normal TM incidence, where $L_{1}=40 \mathrm{~nm}, L_{2}=20 \mathrm{~nm}, t_{1}=280 \mathrm{~nm}, t_{2}=500 \mathrm{~nm}, p=80 \mathrm{~nm}, E_{F 1}=0.55 \mathrm{eV}, E_{F 2}=0.85 \mathrm{eV}$. The white lines denote the outline of GNRs.

On the other hand, there is no obviously enhanced electric field at $k=2167 \mathrm{~cm}^{-1}$ as shown in Figure $3 \mathrm{~b}$. The explanation is that this frequency is far from either of the two resonance dips in the spectral domain.

The widths of GNRs are critical to the plasmonic resonance in MRGB. By gradually adjusting $L_{1}$ from $40 \mathrm{~nm}$ to $16 \mathrm{~nm}$, the first transmission dip can be tuned from $1600 \mathrm{~cm}^{-1}$ to $2550 \mathrm{~cm}^{-1}$ independently as shown in Figure 4a. These two transmission dips tend to merge together as $L_{1}$ becomes smaller and smaller, then MRGB turns out to be a broadband graphene-based biosensor (BGB), which can be used for covering several vibrational lines within a limited frequency range. Similarly, the second transmission dip can also be tuned independently by changing $L_{2}$ as demonstrated in Figure $4 \mathrm{~b}$. The resonant frequency has a blue shift as the width of GNRs decreases, which is mainly attributed to the decrease of the effective length for dipole oscillation along the nanoribbon edges. The natural resonant frequencies of graphene nanoribbons determined by the ribbon width are given by Equation (9) [33,34]:

$$
\omega_{\text {res }} \cong 0.62 \sqrt{\frac{e^{2} \times E_{F}}{\hbar^{2} \varepsilon_{0} \varepsilon_{r} L}}
$$

One can see from Equation (9) that the resonant frequency $\omega_{\text {res }}$ scales with the reciprocal of the square root of the width of graphene nanoribbon.

Another geometric parameter playing a vital role in the MRGB performance is the thickness of dielectric layer $t_{1}$. Figure $4 \mathrm{c}$ depicts that transmission dip will have a dramatic drop with the decrease of $t_{1}$. However, the strength of resonance is also lessened, which impedes the sensitivity of MRGB. Thus, there is an optimal thickness $t_{1}$, at which the performance of MRGB reaches the optimum. The optimal $t_{1}$ is related with the concerning vibration signals. Considering the relatively strong signal strengths for both protein and lipid, $t_{1}$ is chosen as $280 \mathrm{~nm}$ as the optimal value in our simulation.

The discussion above is about normal incidence, the sensing performance of MRGB under oblique incidence is further revealed to satisfy different sensing applications. Transmittance spectra of MRGB covered with protein and lipid molecules under different incident angles are plotted in Figure 4d. As can be seen, two obvious transmission dips remain at around $1600 \mathrm{~cm}^{-1}$ and $2900 \mathrm{~cm}^{-1}$ when $\theta<60^{\circ}$. They overlap with the vibrational fingerprints of corresponding biomolecule in the spectrum. As a consequence, three vertical lines, which indicate the amide-I, amide-II and $\mathrm{CH}_{2}$ absorption bands respectively, line in the regions designated by the dashed lines. Thus, sensing performance of MRGB under oblique incidence shows good robustness, which is significant for optical biosensors. 
(a)

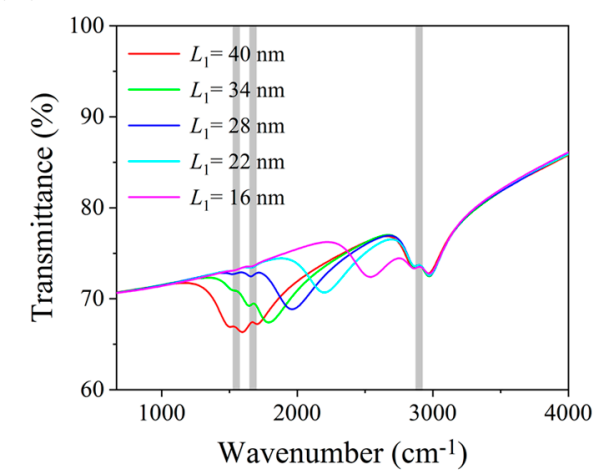

(c)

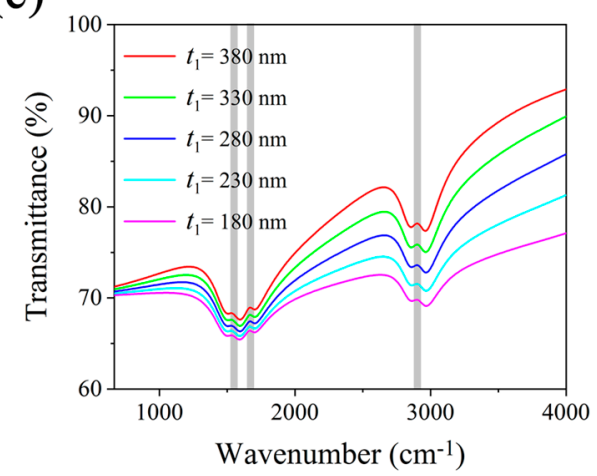

(b)

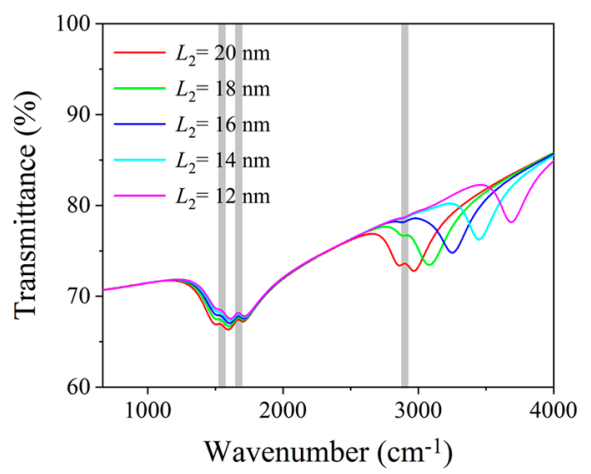

(d)

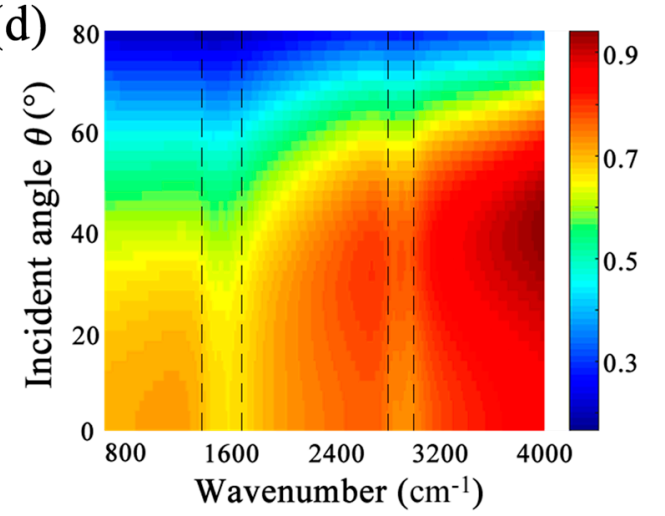

Figure 4. (a-c) Transmittance of MRGB covered with protein and lipid molecules under normal TM incidence, for $p=80 \mathrm{~nm}, t_{2}=500 \mathrm{~nm}, E_{F 1}=0.55 \mathrm{eV}, E_{F 2}=0.85 \mathrm{eV}$. (a) Using different $L_{1}$ values, for $L_{2}=20 \mathrm{~nm}, t_{1}=280 \mathrm{~nm}$. (b) Using different $L_{2}$ values, for $L_{1}=40 \mathrm{~nm}, t_{1}=280 \mathrm{~nm}$. (c) Using different $t_{1}$ values, for $L_{1}=40 \mathrm{~nm}, L_{2}=20 \mathrm{~nm}$. Gray vertical bars represent vibrational bands of protein and lipid molecules. (d) Transmittance as a function of wavenumber and incident angle $\theta$ under TM incidence, where $L_{1}=40 \mathrm{~nm}, L_{2}=20 \mathrm{~nm}, t_{1}=280 \mathrm{~nm}, t_{2}=500 \mathrm{~nm}, p=80 \mathrm{~nm}, E_{F 1}=0.55 \mathrm{eV}$, $E_{F 2}=0.85 \mathrm{eV}$. Dashed vertical black strips outline the vibrational bands of protein and lipid molecules.

The detection limit is another critical figure of merit for a biosensor, which represents how little material it can access. In our simulations, we assume the hybridization of protein and lipid as a homogeneous thin layer covering the entire structure including the antennas and substrate. However, the molecules outside the active area contribute little to the enhanced vibrational signals, since SEIRA signals mainly originate from the molecules located in the antenna hot-spots. Therefore, the exact number of molecules required for detection is hardly to be estimated in the simulations. Nevertheless, to investigate the limit of detection roughly, we vary the thickness of biomolecular layer and tune the resonance to match the vibrational frequency as shown in Figure 5. As can be seen, as the thickness of biomolecular layer decreases from $8 \mathrm{~nm}$ to $2 \mathrm{~nm}$, the enhanced signal strength gets weakened. However, even when the biomolecular layer becomes as thin as $2 \mathrm{~nm}$, corresponding to essentially a single protein monolayer, the enhanced vibrational signal can also be faintly observed. Therefore, the smallest material that the sensor can access is considered to be monolayer molecules. To obtain $I_{\text {SEIRA }}$ in Equation (8), one should iteratively estimate baseline transmittance $T_{\text {baseline }}$ using a smoothing algorithm [35], and then calculate the baseline-corrected vibrational signal $T_{\text {meas }} / T_{\text {baseline, }}$ where $T_{\text {meas }}$ is the simulated transmittance. The signal strength $I_{S E I R A}$ of the enhanced molecular vibrational mode is 0.009 , which is obtained as peak-to-peak value from the baseline-corrected vibrational signal spectrum. The unenhanced signal strength $I_{0}$ is 0.00006 , which can also be obtained by the baseline-corrected algorithm. For the proposed MRGB with GNRs, we assume a finite length of GNRs as $l$ in a single unit cell along the $x$-axis, so $A_{0}$ can be expressed as $A_{0} \approx p \times l$. The enhanced SEIRA signal mainly originates from the molecules located in the GNRs hot-spots as shown in Figure 3. On the basis of this finding, a 
commonly used approximation for the active area is the surface of the GNR edges. Therefore, $A_{\text {SEIRA }}$ can be expressed as $A_{\text {SEIRA }} \approx 2 \Delta \times l(\Delta \ll p)$, where $\Delta$ is the thickness of GNRs. Therefore, when $\Delta$ is assumed as $0.5 \mathrm{~nm}$, by substituting these variables into Equation (8), EF can be obtained as 12,000. Obviously, the approximation is rather rough, but it still provides some guidance for experiments.

(a)

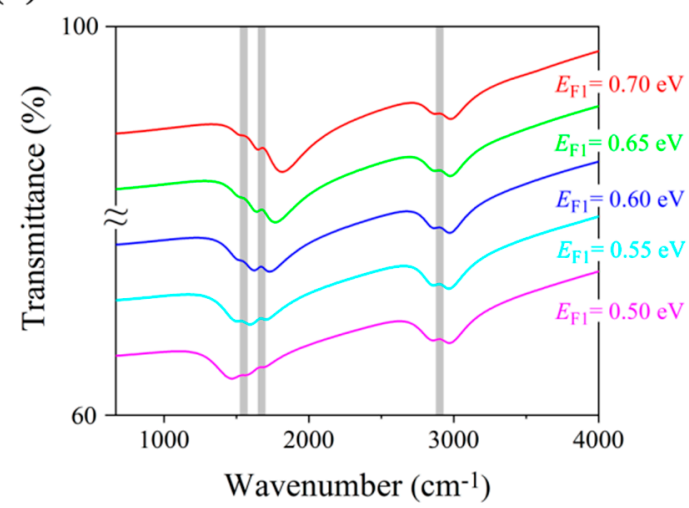

(b)

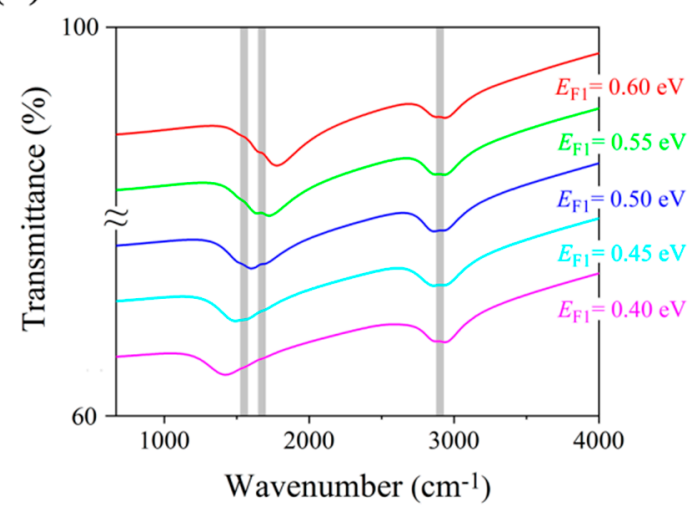

Figure 5. Transmittance spectra of MRGB with different $E_{F 1}$ while accessing different thicknesses of biomolecular layer. (a) For biomolecular layer of $8 \mathrm{~nm}, E_{F 2}=0.85 \mathrm{eV}$. (b) For biomolecular layer of $2 \mathrm{~nm}, E_{F 2}=0.75 \mathrm{eV}$. Other parameters are the same as in Figure 2. Curve with larger $E_{F}$ is translated upward with $6 \%$ in order to demonstrate the spectral shift clearly.

\section{MRGB with Graphene Nanodisks}

GNRs have an infinite length in the $x$-direction compared with the width in the $y$-direction. Thus, the performance of MRGB with GNRs is highly dependent on the polarization of incidence as shown in Figure 2. It can only operate properly under TM polarized incidence, which limits its further application for detection of composite vibrational fingerprints. To overcome the drawback of polarization dependence, we develop an improved three-dimensional (3D) MRGB as illustrated in Figure 6a. A unit of 3D MRGB is made up of two graphene nanodisks (GNDs), $\mathrm{SiO}_{2}$ layers and $\mathrm{Si}$ substrate. Here, the structural variation of the GNDs in the $x$-direction gives the third dimension compared with GNRs. Two nanodisks have different radiuses denoted as $R_{1}$ and $R_{2}$, and we assume $R_{1}>R_{2}$ in the physical model. In the practical applications, the ion-gel layer is commonly considered as a top gate to achieve the tunability of the graphene Fermi energy [36,37]. As plotted in Figure 6a, two sets of interdigitated ion-gel layers with ultra-high capacitance can be spin-coated on top of the proposed MRGB, and two gold gate contacts are fabricated on the interdigitated ion-gel layers to serve as two separated electrodes for the purpose of electrostatic doping. GNDs with different radiuses are controlled by separated top gate voltages, so one can tune Fermi energy of graphene effectively with different bias voltages. The influence on the sensitivity after introducing the ion-gel layer can be neglected due to its ultrathin thickness. The transparent ion-gel can also act as a protective layer to keep GNDs from environmental-induced degradation. 
(a)

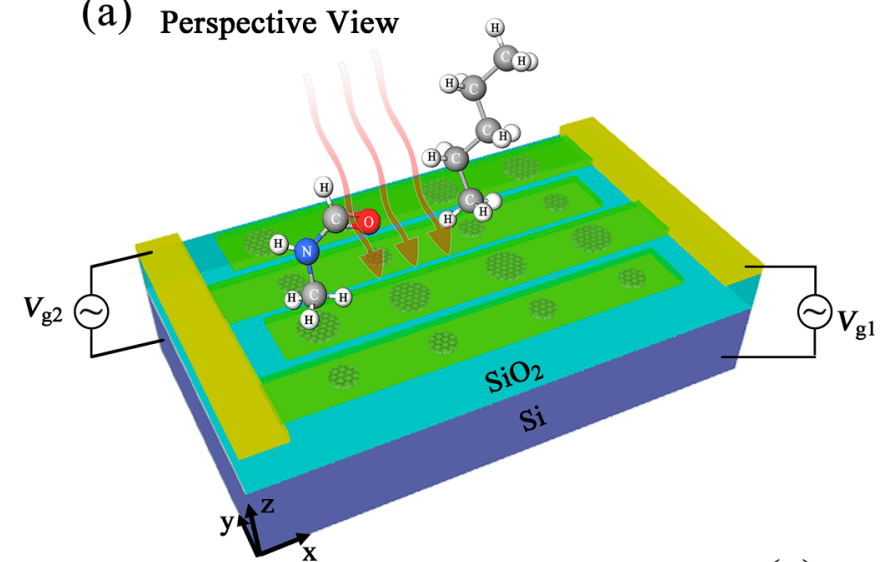

Top View

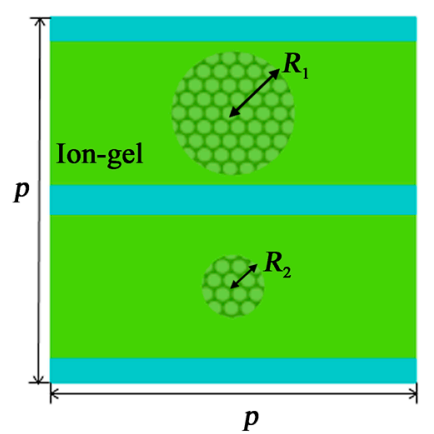

(c) (b)

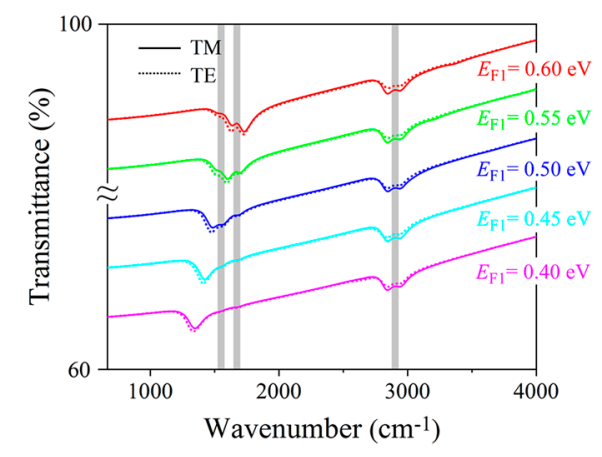

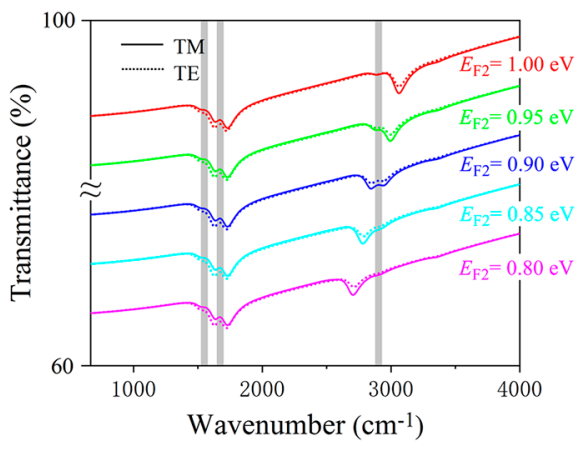

Figure 6. (a) Schematic of the MRGB consisting of periodic graphene nanodisks. $R_{1}$ and $R_{2}\left(R_{1}>R_{2}\right)$ are the radiuses of the bigger and smaller graphene nanodisk, respectively. $(\mathbf{b}, \mathbf{c})$ are the transmittance of $3 \mathrm{D}$ MRGB covered with protein and lipid molecules while sweeping $E_{F 1}(\mathbf{b})$ and $E_{F 2}$ (c) of graphene under normal TM (solid curves) and TE (dashed curves) incidence. Gray vertical bars represent vibrational bands of protein and lipid molecules. Curve with larger $E_{F}$ is translated upward with $6 \%$ in order to demonstrate the spectral shift clearly.

Transmittance spectra of 3D MRGB with protein and lipid under mid-infrared incidence are plotted in Figure $6 b, c$ to investigate the sensing performance for detection of composite vibrational fingerprints in multiple biomolecules. After rigorous optimization for the sensing performance, the geometric parameters are chosen as $R_{1}=20 \mathrm{~nm}, R_{2}=10 \mathrm{~nm}, t_{1}=280 \mathrm{~nm}, t_{2}=500 \mathrm{~nm}$, and $p=80 \mathrm{~nm}$. One can see two obvious transmission dips in the spectra under both TM and TE incidence. In Figure $6 \mathrm{~b}$, when $E_{F 1}=0.40 \mathrm{eV}$, the resonance dip is far from the amide I and II bands, so the vibrational signals of protein are almost undetectable. By continuously varying $E_{F 1}$ from $0.40 \mathrm{eV}$ to $0.60 \mathrm{eV}$, the first transmission dip of MRGB is tuned from $1350 \mathrm{~cm}^{-1}$ to $1700 \mathrm{~cm}^{-1}$, sweeping over the two vibrational fingerprints in protein located at $1668 \mathrm{~cm}^{-1}$ and $1532 \mathrm{~cm}^{-1}$. The molecular vibrational bands of protein are clearly observed due to the increase of spectral overlap. On the other hand, with $E_{F 2}$ fixed at $0.90 \mathrm{eV}$, the second resonant dip remains around $2900 \mathrm{~cm}^{-1}$, resulting in a detection of $\mathrm{CH}_{2}$ band in lipid. In Figure $6 \mathrm{c}$, the first transmission dip is fixed at around $1700 \mathrm{~cm}^{-1}$ with $E_{F 1}$ set as $0.60 \mathrm{eV}$, then the vibrational bands in protein are easily detected attributed to the large spectral overlap. Besides, the second resonance is tuned by bias voltage $V_{g 2}$ to sweep across the lipid vibrational band independently. When $E_{F 2}=0.80 \mathrm{eV}$, the lipid vibrational signal can hardly be detected since it is far from the second transmission dip. As $E_{F 2}$ increases to $0.90 \mathrm{eV}$, the detection of lipid molecules is achieved by enhancing a vibrational signal at $2900 \mathrm{~cm}^{-1}$ corresponding to the $\mathrm{CH}_{2}$ band. If $E_{F 2}$ continues increasing to $1.00 \mathrm{eV}$, the detected lipid vibrational signal almost vanishes as the second resonant dip moves away gradually. Therefore, the proposed 3D MRGB with GNDs is able to identify composite vibrational fingerprints in multiple biomolecules simultaneously for both TM and TE 
polarization. It also possesses the independent tunability for individual plasmonic resonance to satisfy different sensing applications.

Next, we further investigate the sensing performance of 3D MRGB under non-normal mid-infrared incidence. In Figure 7a, transmittance spectra of 3D MRGB with protein and lipid as a function of wavenumber and incident angle $\theta$ are depicted for TM polarization. The parameters are $R_{1}=20 \mathrm{~nm}$, $R_{2}=10 \mathrm{~nm}, t_{1}=280 \mathrm{~nm}, t_{2}=500 \mathrm{~nm}, p=80 \mathrm{~nm}, E_{F 1}=0.6 \mathrm{eV}, E_{F 2}=0.9 \mathrm{eV}$. When $\theta$ is below $72^{\circ}$, protein can be detected due to the spectral overlapping between the vibrational fingerprints and the first resonance. The vibrational fingerprint of lipid is also enhanced to some extent by the second resonance. When $\theta$ increases to $68^{\circ}$, the protein molecules can still be identified while the signal of lipid molecules is too weak to detect. In Figure $7 \mathrm{~b}$, for TE polarization, the vibrational fingerprints of protein is clearly detected until the incident angle is up to $70^{\circ}$. The lipid can be detected with a faint signal, and is almost undetectable when $\theta$ increases up to $65^{\circ}$. Therefore, the $3 \mathrm{D}$ MRGB can guarantee sensing ability in a wide incident angle range for both TM and TE polarized incidence.

(a)

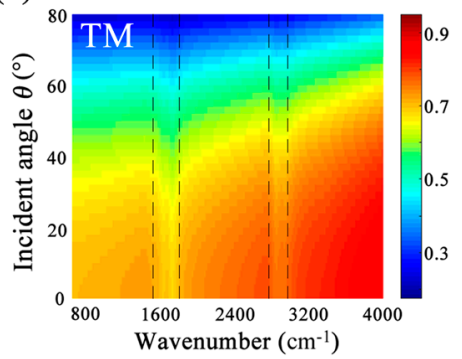

(b)

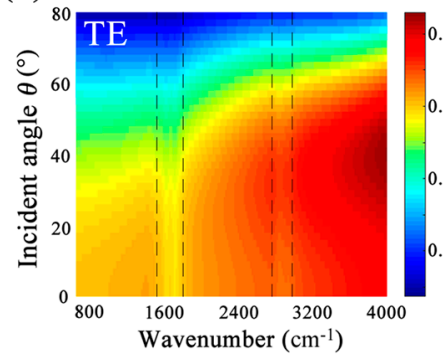

(c)

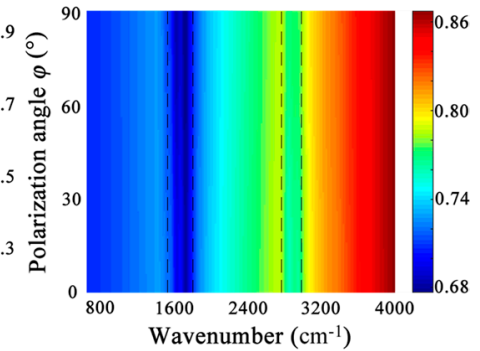

Figure 7. Transmittance of 3D MRGB covered with protein and lipid molecules as functions of wavenumber and incident angle $\theta$ of TM (a) and TE (b) polarization, as well as wavenumber and the polarization angle $\varphi$ under normal incidence (c). Dashed vertical black strips outline the vibrational bands of protein and lipid molecules.

Finally, the polarization dependence of 3D MRGB for detecting protein and lipid molecules is further evaluated. The transmission spectra under normal incidence with polarization angle $\varphi$ from $0^{\circ}$ to $90^{\circ}$ are shown in Figure $7 \mathrm{c}$. We assume the polarization angle of $\mathrm{TM}$ incidence to be $0^{\circ}$, so the transmittance in Figure 7c at $\varphi=0^{\circ}$ is the same as the solid curves plotted in Figure 6b or Figure 6c. As $\varphi$ increases to $90^{\circ}$, the incidence slightly becomes a TE polarized mid-infrared light. Therefore, the transmittance gradually turns out to be the same as the dashed curves in Figure $6 \mathrm{~b}$ or Figure $6 \mathrm{c}$, which is a little different from that of TM polarization. When $0^{\circ}<\varphi<90^{\circ}$, both $x$-axis and $y$-axis components coexist in the incident electric field, so the transmittance is shown as a non-polarized status. The vibrational fingerprints of protein are clearly observed at any polarization angle, while that of lipid is slightly weakened as the polarization angle increases. However, even if the polarization is up to $90^{\circ}$ (TE polarization), a tiny vibrational signal located at the $\mathrm{CH}_{2}$ band can also be detected as shown in Figure $6 \mathrm{~b}, \mathrm{c}$. When $E_{F 1}$ is fixed at $0.60 \mathrm{eV}$ and $E_{F 2}$ is $0.90 \mathrm{eV}$, for TM polarization, the transmittance rates at $1532 \mathrm{~cm}^{-1}$ (amide-I), $1668 \mathrm{~cm}^{-1}$ (amide-II) and $2900 \mathrm{~cm}^{-1}\left(\mathrm{CH}_{2}\right)$ are $71 \%, 69 \%$ and $78 \%$, respectively, while they are $72 \%, 70 \%$ and $77 \%$ for TE polarization. The small difference of sensing performance between TM and TE polarization is mainly because the unit cell of 3D MRGB is not four-fold symmetrical, although both the big GNDs and small GNDs can support plasmonic localized resonance in either $x$-axis or $y$-axis direction.

\section{Conclusions}

In summary, we have numerically designed a label-free MRGB with periodic GNRs of which each resonance band can be independently tuned by electrostatic gating. The decent detection performance for vibrational fingerprints in protein and lipid is revealed due to the tunable spectral selectivity and extreme field confinement of graphene. Electric field distributions are plotted to demonstrate the 
hotspots and investigate the physical mechanism. Geometric parameters are critical for the performance of MRGB and the robustness for oblique incidence is also elaborated. In addition, a MRGB with periodic GNDs is further developed to solve the issue of polarization dependence. We believe that the design principles in this paper will provide a significant guide in designing other multi-resonant SEIRA sensors based on graphene.

Author Contributions: Y.C. and Y.H. conceived the research, undertook the simulations and analysis, and wrote the manuscript. X.W., J.Z., J.Y. and Y.Z. contributed to the analysis and the manuscript review.

Funding: This work is supported by the fund of Key Laboratory of THz Technology, Ministry of Education, China; NSAF (No. U1830116); Scientific Research Climbing Plan of Xiamen University of Technology (No. XPDKQ18025).

Conflicts of Interest: The authors declare no conflicts of interest.

\section{References}

1. Liedberg, B.; Nylander, C.; Lundstrom, I. Biosensing with surface plasmon resonance-How it all started. Biosens. Bioelectron. 1995, 10, i-ix. [CrossRef]

2. Rich, R.L.; Myszka, D.G. Advances in surface plasmon resonance biosensor analysis. Curr. Opin. Biotechnol. 2000, 11, 54-61. [CrossRef]

3. Neubrech, F.; Pucci, A.; Cornelius, T.W.; Karim, S.; Etxarri, A.G.; Aizpurua, J. Resonant plasmonic and vibrational coupling in a tailored nanoantennas for infrared detection. Phys. Rev. Lett. 2008, 101, 157403. [CrossRef] [PubMed]

4. Neubrech, F.; Huck, C.; Weber, K.; Pucci, A.; Giessen, H. Surface-enhanced infrared spectroscopy using resonant nanoantennas. Chem. Rev. 2017, 117, 5110-5145. [CrossRef]

5. Wu, C.; Khanikaev, A.B.; Adato, R.; Arju, N.; Yanik, A.A.; Altug, H.; Shvets, G. Fano-Resonant asymmetric metamaterials for ultrasensitive spectroscopy and identification of molecular monolayers. Nat. Mater. 2011, 11, 69-75. [CrossRef]

6. Dong, L.; Yang, X.; Chao, Z.; Cerjan, B.; Zhou, L.; Tseng, M.L.; Zhang, Y.; Alabastri, A.; Nordlander, P.; Halas, N.J. Nanogapped Au antennas for ultrasensitive surface-enhanced infrared absorption spectroscopy. Nano Lett. 2016, 17, 5768-5774. [CrossRef]

7. Braun, A.; Maier, S.A. Versatile direct laser writing lithography technique for surface-enhanced infrared spectroscopy sensors. ACS Sens. 2016, 1, 1155-1162. [CrossRef]

8. Chen, K.; Adato, R.; Altug, H. Dual-band perfect absorber for multispectral plasmon-enhanced. ACS Nano 2012, 6, 7798-8006. [CrossRef]

9. Aouani, H.; Rahmani, M.; Sipova, H.; Torres, V.; Hegnerova, K.; Beruete, M.; Homola, J.; Hong, M.; Cia, M.N.; Maier, S.A. Plasmonic nanoantennas for multispectral surface-enhanced spectroscopies. J. Phys. Chem. C 2013, 117, 18620-18626. [CrossRef]

10. Rodrigo, D.; Tittle, A.; Herpin, A.J.; Limaj, O.; Altug, H. Self-similar multi-resonant nanoantennas arrays for sensing from near- to mid-infrared. ACS Photonics 2018, 5, 4903-4911. [CrossRef]

11. Rodrigo, D.; Tittle, A.; Bouziad, N.A.; Herpin, A.J.; Limaj, O.; Kelly, C.; Yoo, D.; Wittenberg, N.J.; Oh, S.H.; Lashuel, H.A.; et al. Resolving molecule-specific information in dynamic lipid membrane processes with multi-resonant infrared metasurfaces. Nat. Commun. 2018, 9, 2160. [CrossRef] [PubMed]

12. Tittl, A.; Leitis, A.; Liu, M.; Yesilkoy, F.; Choi, D.Y.; Neshev, D.N.; Kivshar, Y.S.; Altug, H. Imaging-based molecular barcoding with pixelated dielectric metasurfaces. Science 2018, 360, 1105-1109. [CrossRef] [PubMed]

13. Novoselov, K.S.; Geim, A.K.; Morozov, S.V.; Jiang, D.; Zhang, Y.; Dubonos, S.V.; Grigorieva, I.V.; Firsov, A.A. Electric field effect in atomically thin carbon films. Science 2004, 306, 666-669. [CrossRef] [PubMed]

14. Geim, A.K.; Novoselov, K.S. The rise of graphene. Nat. Mater. 2007, 6, 183-191. [CrossRef]

15. Cai, Y.; Zhu, J.; Liu, Q.H. Tunable enhanced optical absorption of graphene using plasmonic perfect absorbers. Appl. Phys. Lett. 2015, 106, 043105. [CrossRef]

16. Xiao, S.; Wang, T.; Liu, Y.; Xu, C.; Han, X.; Yan, X. Tunable light trapping and absorption enhancement with graphene ring arrays. Phys. Chem. Chem. Phys. 2016, 18, 26661-26669. [CrossRef]

17. Li, H.; Ji, C.; Ren, Y.; Hu, J.; Qin, M.; Wang, L. Investigation of multiband plasmonic metamaterial perfect absorbers based on graphene ribbons by the phase-coupled method. Carbon 2019, 141, 481-487. [CrossRef] 
18. Yasa, E.; Arif, E.C.; Habibe, D. Multi-band plasmonic platform utilizing UT-shaped graphene antenna arrays. Plasmonic 2018, 13, 1081-1088.

19. Li, Y.; Yan, H.; Farmer, D.B.; Meng, X.; Zhu, W.; Osgood, R.M.; Heinz, T.F.; Avouris, P. Graphene plasmon enhanced vibrational sensing of surface-adsorbred layer. Nano Lett. 2014, 14, 1573-1577. [CrossRef]

20. Farmer, D.B.; Avouris, P.; Li, Y.; Heinz, T.F.; Han, S. Ultrasensitive plasmonic detection of molecules with graphene. ACS Nano 2016, 3, 553-557. [CrossRef]

21. Rodrigo, D.; Limaj, O.; Janner, D.; Etezadi, D.; De Abajo, F.J.G.; Pruneri, V.; Altug, H. Mid-infrared plasmonic biosensing with graphene. Science 2015, 349, 165-168. [CrossRef] [PubMed]

22. Hu, H.; Yang, X.; Zhai, F.; Hu, D.; Liu, R.; Liu, K.; Sun, Z.; Dai, Q. Far-field nanoscale infrared spectroscopy of vibrational fingerprints of molecules with graphene plasmons. Nat. Commun. 2016, 7, 1-8. [CrossRef] [PubMed]

23. Hu, H.; Yang, X.; Guo, X.; Khaliji, K.; Biswas, S.R.; De Abajo, F.J.G.; Low, T.; Sun, Z.; Dai, Q. Gas identification with graphene plasmons. Nat. Commun. 2019, 10, 1131. [CrossRef] [PubMed]

24. Zhu, Y.; Li, Z.; Hao, Z.; DiMarco, C.; Maturavongsadit, P.; Hao, Y.; Lu, M.; Stein, A.; Wang, Q.; Hone, J.; et al. Optical conductivity-based ultrasensitive mid-infrared biosensing on a hybrid metasurface. Light Sci. Appl. 2018, 7, 67. [CrossRef] [PubMed]

25. Palik, E.D. Handbook of Optical Constants of Solids, 3rd ed.; Academic Press: San Diego, CA, USA, 1998; pp. 155-187.

26. Zhu, J.; Cheng, J.; Zhang, L.; Liu, Q.H. Modeling of 2D graphene material for plasmonic hybrid waveguide with enhanced near-infrared modulation. Mater. Lett. 2017, 186, 53-56. [CrossRef]

27. Hanson, G.W. Dyadic Green's functions and guided surface waves for a surface conductivity model of graphene. J. Appl. Phys. 2008, 103, 064302. [CrossRef]

28. Gómez-Díaz, J.S.; Perruisseau-Carrier, J. Graphene-based plasmonic switches at near infrared frequencies. Opt. Express 2013, 21, 15490-15504. [CrossRef]

29. Picard, F.; Buffeteau, T.; Desbat, B.; Auger, M.; Pezolet, M. Quantitative orientation measurements in thin lipid films by attenuated total reflection infrared spectroscopy. Biophys. J. 1999, 76, 539-551. [CrossRef]

30. Adato, R.; Artar, A.; Erramilli, S.; Altug, H. Engineered Absorption Enhancement and Induced Transparency in Coupled Molecular and Plasmonic Resonator Systems. Nano Lett. 2013, 13, 2584-2591. [CrossRef]

31. Cai, Y.; Xu, K.D. Tunable broadband terahertz absorber based on multilayer graphene-sandwiched plasmonic structure. Opt. Express 2018, 26, 31693-31705. [CrossRef]

32. Cai, Y.; Xu, K.D.; Feng, N.; Guo, R.; Lin, H.; Zhu, J. Anisotropic infrared plasmonic broadband absorber based on graphene-black phosphorus multilayers. Opt. Express 2019, 27, 3101-3112. [CrossRef] [PubMed]

33. Ju, L.; Geng, B.S.; Horng, J.; Girit, C.; Martin, M.; Hao, Z.; Bechtel, H.A.; Liang, X.G.; Zettl, A.; Shen, Y.R.; et al. Graphene plasmonics for tunable terahertz metamaterials. Nat. Nanotechnol. 2011, 6, 630-634. [CrossRef] [PubMed]

34. Gao, W.; Shu, J.; Qiu, C.; Xu, Q. Excitation of plasmonic waves in graphene by guided-mode resonances. ACS Nano 2012, 6, 7806-7813. [CrossRef] [PubMed]

35. Eilers, P.H.C. A Perfect Smoother. Anal. Chem. 2003, 75, 3631-3636. [CrossRef] [PubMed]

36. Fang, Z.Y.; Wang, Y.; Schlather, A.E.; Liu, Z.; Ajayan, P.M.; Javier García de Abajo, F.; Nordlander, P.; Zhu, X.; Halas, N.J. Active tunable absorption enhancement with graphene nanodisk arrays. Nano Lett. 2014, 14, 299-304. [CrossRef] [PubMed]

37. Fang, Z.Y.; Thongrattanasiri, S.; Schlather, A.; Liu, Z.; Ma, L.; Wang, Y.; Ajayan, P.M.; Nordlander, P.; Halas, N.J.; Javier García de Abajo, F. Gated tunability and hybridization of localized plasmons in nanostructured graphene. ACS Nano 2013, 7, 2388-2395. [CrossRef] [PubMed]

(C) 2019 by the authors. Licensee MDPI, Basel, Switzerland. This article is an open access article distributed under the terms and conditions of the Creative Commons Attribution (CC BY) license (http://creativecommons.org/licenses/by/4.0/). 